\title{
Prospects for probing strong gravity with a pulsar-black hole system
}

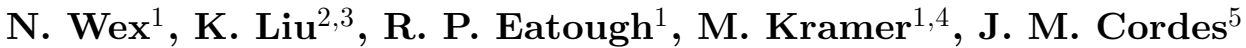 \\ and T. J. W. Lazio ${ }^{6} \dagger$ \\ ${ }^{1}$ Max-Planck-Institut für Radioastronomie, Auf dem Hügel 69, D-53121 Bonn, Germany \\ email: wex@mpifr.de \\ ${ }^{2}$ Laboratoire de Physique et Chimie de l'Environnement, LPCE UMR 6115 CNRS, \\ F-45071 Orleans Cedex 02, France \\ ${ }^{3}$ Station de radioastronomie de Nancay, Observatoire de Paris, CNRS/INSU, \\ F-18330 Nancay, France \\ ${ }^{4}$ University of Manchester, Jodrell Bank Centre for Astrophysics, Alan-Turing Building, \\ Manchester M13 9PL, UK \\ ${ }^{5}$ Astronomy Dept., Cornell Univ., Ithaca, NY 14853, USA \\ ${ }^{6}$ Jet Propulsion Laboratory, California Institute of Technology, M/S 138-308, \\ 4800 Oak Grove Dr., Pasadena, CA 91109, USA
}

\begin{abstract}
The discovery of a pulsar (PSR) in orbit around a black hole (BH) is expected to provide a superb new probe of relativistic gravity and $\mathrm{BH}$ properties. Apart from a precise mass measurement for the $\mathrm{BH}$, one could expect a clean verification of the dragging of space-time caused by the $\mathrm{BH}$ spin. In order to measure the quadrupole moment of the $\mathrm{BH}$ for testing the no-hair theorem of general relativity (GR), one has to hope for a sufficiently massive BH. In this respect, a PSR orbiting the super-massive BH in the center of our Galaxy would be the ultimate laboratory for gravity tests with PSRs. But even for gravity theories that predict the same properties for BHs as GR, a PSR-BH system would constitute an excellent test system, due to the high grade of asymmetry in the strong field properties of these two components. Here we highlight some of the potential gravity tests that one could expect from different PSR-BH systems, utilizing present and future radio telescopes, like FAST and SKA.
\end{abstract}

Keywords. pulsars: general, black hole physics, relativity

In the following we summarize the work presented in Liu et al. (2012), Liu (2012) and Liu et al. (in prep.). Based on these publications, we will highlight three different aspects of testing gravity with a PSR-BH system:

- Testing gravity with a PSR in orbit with a stellar mass $\left(\sim 10 M_{\odot}\right) \mathrm{BH}$.

- Testing gravity with a PSR in orbit around the BH in the Galactic center, Sgr A*.

- Testing scalar-tensor gravity with a PSR-BH system, as an example for the probing power of a PSR-BH system for gravity theories, in which BHs are the same as in GR.

The results given are based on extensive mock data simulations and consistent timing models, which are explained in detail in Wex \& Kopeikin (1999), Liu et al. (2012), and Liu (2012). Furthermore, these simulations have been conducted for three different types of radio telescopes: a 100-m class telescope, FAST and SKA. A detailed discussion on the expected timing precision for these telescopes can be found in Liu et al. (2012) and Liu (2012).

$\dagger$ Part of this research was carried out at the Jet Propulsion Laboratory, California Institute of Technology, under a contract with the National Aeronautics and Space Administration. 


\section{Black hole properties}

One of the most intriguing results of general relativity (GR) is the uniqueness theorem for the stationary BH solutions of the Einstein-Maxwell equations (see Chruściel et al. 2012, and references therein). It implies that in GR all uncharged BH solutions are described by the Kerr metric and, therefore, uniquely determined by mass $M$ and spin $S$. Astrophysical BHs are believed to be the result of a gravitational collapse, during which all the properties of the progenitor, apart from mass and spin, are radiated away by gravitational radiation (Price 1972a,b). The outer spacetime of an astrophysical BH should therefore (to a very good approximation) be described by the Kerr metric. Since the Kerr metric has a maximum spin at which it still exhibits an event horizon, Penrose's cosmic censorship conjecture (CCC) within GR (Penrose 1979) requires the dimensionless spin parameter $\chi$ to satisfy

$$
\chi \equiv \frac{c}{G} \frac{S}{M^{2}} \leqslant 1
$$

A measured value for $\chi$ that exceeds 1 would pose a serious problem for our understanding of spacetime, since this would indicate that either GR is wrong or that a region may be visible to the outside universe, where our present understanding of gravity and spacetime breaks down.

As a result of the no-hair theorem, all higher multipole moments $(l \geqslant 2)$ of the gravitational field of an astrophysical BH can be expressed as a function of $M$ and $S$ (Hansen 1974). In particular, the dimensionless quadrupole moment $q$ satisfies the relation

$$
q \equiv \frac{c^{4}}{G^{2}} \frac{Q}{M^{3}}=-\chi^{2}
$$

A measurement of the quadrupole moment, in combination with a mass and a spin measurement, would therefore identify the $\mathrm{BH}$ as a Kerr $\mathrm{BH}$ and provide a test of the no-hair theorem for BHs.

\section{A pulsar in orbit with a stellar-mass black hole}

Concerning possible formation scenarios of PSR-BH systems, a detailed discussion is presented in Liu (2012) and Liu et al. (in prep.). For the following, it is only important to notice that there are several formation scenarios that lead to a PSR-BH system with a recycled PSR.

\subsection{Mass determination}

The most precise mass measurements for stars (other than the Sun) come from PSR timing observations (Lorimer \& Kramer 2005, Weisberg et al. 2010). Those are achieved in binary PSR systems, where in addition to the Keplerian parameters one can determine a set of post-Keplerian $(\mathrm{PK})$ parameters that describe the relativistic correction in the orbital motion and signal propagation. In GR (and many alternative theories of gravity) the PK parameters are functions of the Keplerian parameters and the two a priori unknown masses of the system, which can be determined once two PK parameters have been obtained (Lorimer \& Kramer 2005). In a PSR-BH system the observation of PK parameters will allow the determination of the $\mathrm{BH}$ mass with unprecedented precision. Fig. 1 (taken from Liu et al., in prep.) illustrates the precision that one can expect with a 100-m class telescope. Future telescopes like FAST and SKA would yield a significantly higher precision, as demonstrated by the simulations in Liu (2012) and Liu et al. (in prep.). 


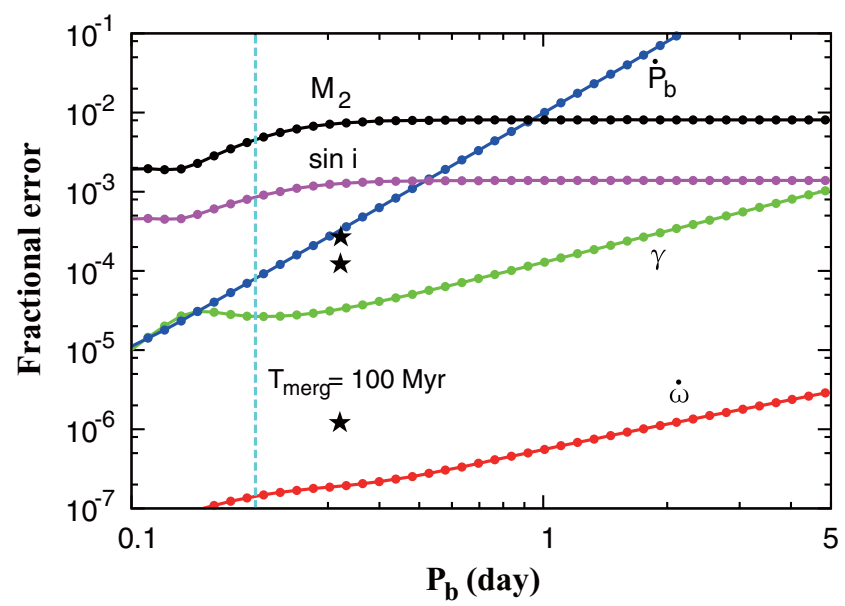

Figure 1. Fractional error for different PK parameters, which can be used to determine the mass of the $\mathrm{BH}$, as a function of the orbital period. The figure is based on mock data simulations for weekly observations over 5 years with a 100-m class telescope, assuming a recycled PSR in a mildly eccentric $(e=0.1)$ orbit with a $10 M_{\odot} \mathrm{BH}$. Note that $\dot{\omega}$ is expected to have a significant contribution from the frame-dragging caused by the rotation of the $\mathrm{BH}$, and can a priori not be used in the mass determination. For a comparison, the three stars mark the precision obtained in the Hulse-Taylor pulsar (top to bottom: $\dot{P}_{b}, \gamma, \dot{\omega}$; Weisberg et al. 2010).

\subsection{Frame dragging}

It has been shown by Wex \& Kopeikin (1999) that the Lense-Thirring precession of the orbit, caused by the relativistic spin-orbit coupling, between the orbital angular momentum and the $\mathrm{BH}$ spin, is the best way to measure the magnitude and direction of the $\mathrm{BH}$ spin. Once the second time derivatives of the longitude of periastron and the projected semi-major axis become observable in the timing data, the spin can be determined. Fig. 2 (taken from Liu et al., in prep.) illustrates the precision that one can expect with SKA. A spin measurement in a PSR-BH system would verify the frame dragging caused by a rotating $\mathrm{BH}$, and test the $\mathrm{CCC}$ inequality $\chi \leqslant 1$.

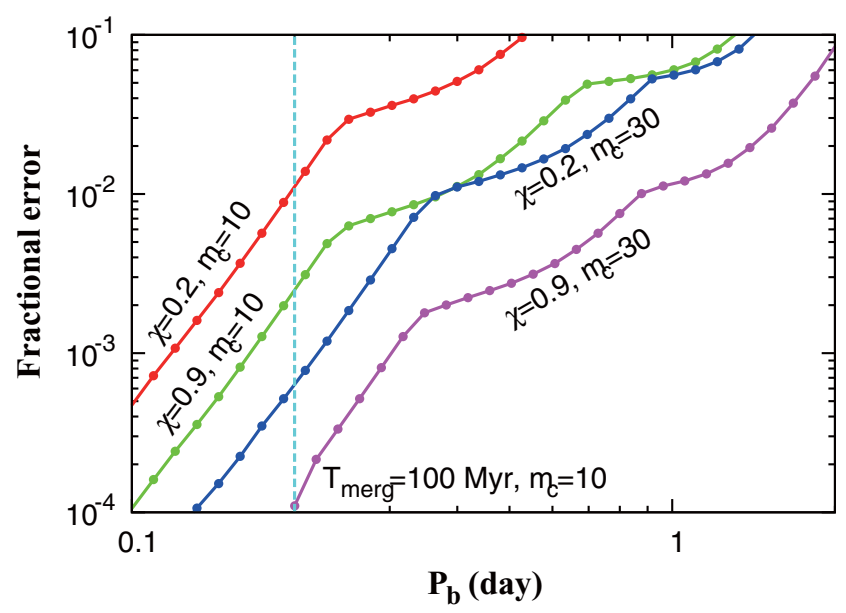

Figure 2. Fractional error in the BH spin, as a function of the orbital period. The figure is based on mock data simulations for weekly observations over 5 years with SKA, assuming a recycled PSR in a mildly eccentric $(e=0.1)$ orbit. $m_{c}$ denotes the $\mathrm{BH}$ mass. 


\subsection{Quadrupole moment}

In order to perform a test of the no-hair theorem, it would be necessary to measure the quadrupole moment of the BH. As already argued by Wex \& Kopeikin (1999), such a measurement has to be based on the periodic features in the orbital motion, caused by the unique structure of the gravitational potential associated with the quadrupole moment. Our simulations, presented in Liu (2012) and Liu et al. (in prep.) show, that only for a very massive $\mathrm{BH}\left(\gtrsim 30 M_{\odot}\right)$ and very tight orbits $\left(P_{b} \lesssim 0.2 \mathrm{~d}\right)$ one can hope to measure the quadrupole signature in the data, provided one has the superb timing precision of the SKA. We consider it as not very likely, that such a system can be found in nature, in particular since such a system would have a very short lifetime in the order of a few Myr.

\section{A pulsar in orbit around Sgr $\mathbf{A}^{*}$}

According to the previous section, a system consisting of a radio PSR and a stellar mass $\mathrm{BH}$ will most likely not allow the determination of the $\mathrm{BH}$ quadrupole moment to test the no-hair theorem. Since the quadrupole moment becomes more important for more massive BHs, one wishes to find a PSR in orbit around the most massive BH in our Galaxy, Sgr A* in the Galactic center $\left(M \sim 4 \times 10^{6} M_{\odot}\right.$; Ghez et al. 2008, Gillessen et al. 2009). Finding and timing a PSR in orbit around Sgr A* comes with certain challenges, which are discussed in more details in Liu et al. (2012), Eatough et al. (this proceedings) and references therein. In short, it is more likely to detect a young PSR with a rotational period $\sim 0.5 \mathrm{~s}$, which then would give us a timing precision in the order of $\sim 0.1$ to $1 \mathrm{~ms}$. All this requires observational frequencies $\gtrsim 20 \mathrm{GHz}$. Furthermore, one would also have to worry about perturbations by other masses in the immediate vicinity of Sgr A* (see Liu et al. 2012, and references therein). In the results presented here we assume a clean orbit and a weekly time-of-arrival measurement with $0.1 \mathrm{~ms}$ uncertainty. As we will see, this is already sufficient, to go all the way to the no-hair theorem test, i.e. measuring the

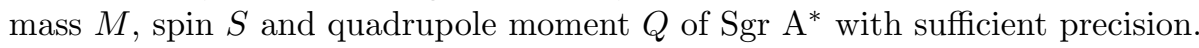

\subsection{Mass determination and the distance to the Galactic center}

For the precision that can be obtained in determining the mass of Sgr $\mathrm{A}^{*}$ from PK parameters, the PSR can be seen as a test mass in orbit around Sgr A*. In this case the measurement of one PK parameter is sufficient. A fractional precision of $10^{-5}$, or even better, should be easily possible with a PSR in a $\lesssim 1$ yr orbit (Liu et al. 2012). Such a precision is not only key to extract the Lense-Thirring contribution to the peri-center precession $\dot{\omega}$, it would also allow, in combination with astrometric observations of the S-stars, to determine the distance to the Galactic center $R_{0}$, since, unlike the astrometric mass, the mass measurement from PSR timing is not affected by an uncertainty in $R_{0} \cdot \dagger$

\subsection{Frame dragging}

Although there is clear indication that $\mathrm{Sgr} \mathrm{A}^{*}$ rotates, its actual rate of rotation is still not well determined, and a rather large range in the estimates of $\chi$ can be found in the literature (see references in Liu et al. 2012), which is a result of the uncertainty in the underlying model assumptions. A PSR would, in the absence of any major external perturbations, give direct access to the dragging of inertial frames in the vicinity of Sgr $\mathrm{A}^{*}$. Like in the case of stellar mass BHs, the (additional) precession of a PSR orbit due to the

$\dagger$ According to our estimates, in combination with a $10 \mu$ as infrared astrometry, an uncertainty of just a few pc could be reached. 
frame dragging (Lense-Thirring precession) is the most promising effect to determine the direction and magnitude of the BH spin. Liu et al. (2012) have used extensive mock data simulations to show, that for orbits below an orbital period of 0.5 years, where external perturbations are likely to be negligible, the spin parameter $\chi$ could be measured with a precision of $10^{-4}$ to $10^{-3}$, or even better in case of very short orbital periods $\left(P_{b} \lesssim 0.1 \mathrm{yr}\right)$ and/or high eccentricities $(e \gtrsim 0.8)$. This would be a test of the frame dragging caused by a super-massive $\mathrm{BH}$ and, like in section 2.2 , a test of the CCC inequality $\chi \leqslant 1$.

\subsection{No-hair theorem test}

For a $\mathrm{BH}$, with a given spin parameter $\chi$, the quadrupole moment grows very fast with increasing mass $\left(\propto M^{3}\right)$. For this reason, even for rather wide orbits of several $10 \mathrm{AU}$ the quadrupole moment of Sgr $\mathrm{A}^{*}$ is expected to give rise to measurable effects in the orbital motion. The calculations in Liu et al. (2012) clearly show, that the observable amplitudes of the "quadrupole effect", for an orbit of a few $0.1 \mathrm{yr}$, are of order of several milli-seconds, and should allow a better than $1 \%$ test of the no-hair theorem. In contrast to an astrometric no-hair theorem test (Will 2008), only one star (here the PSR) is needed and the orbital eccentricity can be considerably less extreme (see Fig. 10 in Liu et al. 2012).

\section{Scalar-tensor gravity and PSR-BH systems}

In the previous sections we have seen the potential to probe the properties of gravity with a PSR in orbit around a BH. But even for gravity theories that predict the same properties for a $\mathrm{BH}$ as GR, a PSR-BH system would be an excellent probe, that can be superior to all present binary PSR experiments. An important class of gravity theories where this is the case, as pointed out by Damour \& Esposito-Farèse (1998), are scalartensor theories. Scalar-tensor gravity, in which gravity is mediated by a tensor field $g_{\mu \nu}^{*}$ and by a massless scalar field $\varphi$, are well motivated and physically consistent alternatives to GR, that have been studied extensively (see Fujii \& Maeda 2003). In the scalar-tensor gravity, investigated in detail in Damour \& Esposito-Farèse (1996), the orbital motion of a binary system depends, besides the Einstein masses $m_{A}, m_{B}$ on the effective coupling constants $\alpha_{a}=\partial \ln m_{a} / \partial \varphi_{0}(a=A, B)$, and their scalar-field derivatives $\partial \beta_{a} / \partial \varphi_{0}$, where $\varphi_{0}$ denotes the asymptotic value of $\varphi$ at spatial infinity. In a PSR-BH system, we have $\alpha_{\mathrm{BH}}=0$ (and consequently $\beta_{\mathrm{BH}}=0$ ) because of the no-scalar-hair theorems, which considerably simplifies the equations for the PK parameters given in Damour \& Esposito-Farèse (1996). Of particular interest here is the leading term in the gravitational wave damping (dipolar radiation) of the orbital period $P_{b}$, that is simply

$$
\dot{P}_{b}^{\text {dipolar }} \simeq-\frac{4 \pi^{2} G}{c^{3} P_{b}} \frac{m_{\mathrm{PSR}} m_{\mathrm{BH}}}{m_{\mathrm{PSR}}+m_{\mathrm{BH}}} \frac{1+e^{2} / 2}{\left(1-e^{2}\right)^{5 / 2}} \alpha_{\mathrm{PSR}}^{2} .
$$

For a given equation-of-state, the effective scalar coupling of the PSR, $\alpha_{\mathrm{PSR}}$, depends on the two fundamental coupling parameters of the theory, $\alpha_{0}$ (linear) and $\beta_{0}$ (quadratic), and the mass of the PSR, which we assume to be the canonical value of $1.4 M_{\odot}$. Liu et al. (in prep.) have conducted extensive mock data simulations for different types of telescopes and different orbital parameters to demonstrate the superb capabilities of a PSR-BH binary to constrain scalar-tensor gravity. Figure 3 presents some of these results. As can be seen there, in particular with future radio telescopes a PSR-BH system would provide an extremely sensitive test for the presence of a scalar degree of freedom in the gravitational interaction. 


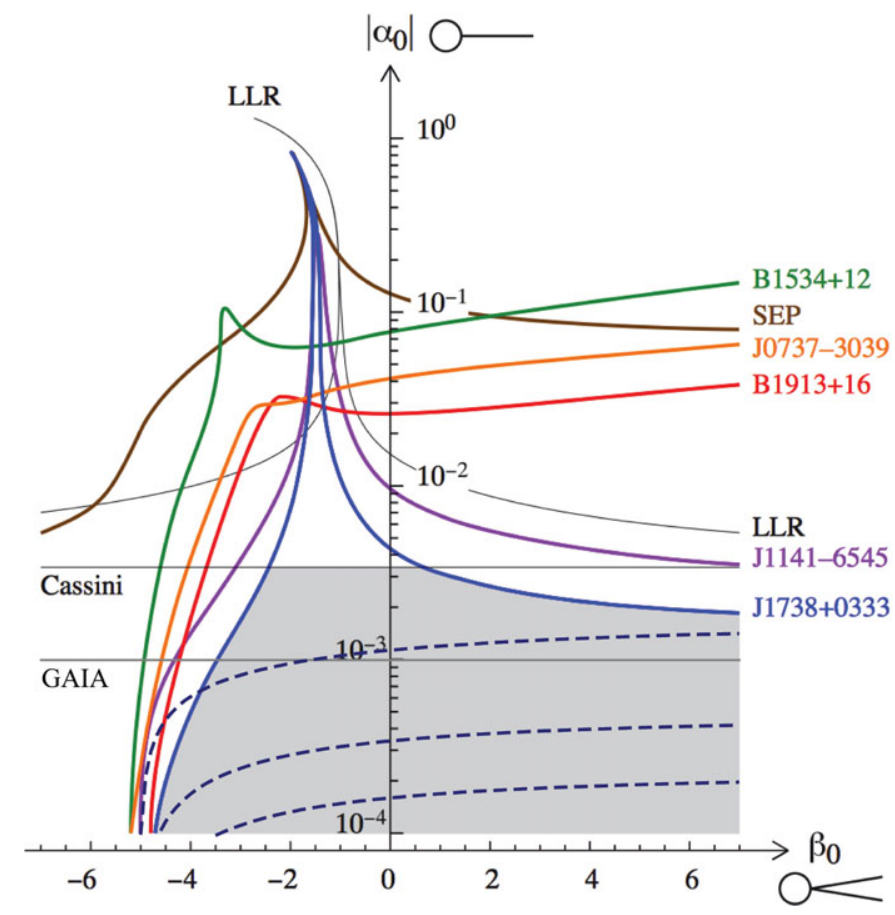

Figure 3. Constraints in the $\beta_{0}-\alpha_{0}$ plane (different PSRs and a hypothetical PSR-BH system). Details are given in Freire et al. (2012) and Liu et al. (in prep.). Limits by a hypothetical PSR-BH system, consisting of a recycled PSR in orbit with a $10 M_{\odot} \mathrm{BH}\left(P_{b}=5 \mathrm{~d}, e=0.8\right)$ are given by dashed lines, which exclude the region above and left of them. The three lines, correspond to (top to bottom): $10 \mathrm{yr}$ weekly timing with a $100-\mathrm{m}$ class telescope, $5 \mathrm{yr}$ weekly timing with FAST, and $5 \mathrm{yr}$ weekly timing with SKA.

\section{References}

Chruściel, P. T., Costa, J. L., \& Heusler, M., 2012 "Stationary Black Holes: Uniqueness and Beyond", Living Rev. Relativity, 15, 7. URL (cited on 15. Oct. 2012): http://www.livingreviews.org/lrr-2012-7

Damour, T., \& Esposito-Farèse, G., 1996, Phys. Rev. D, 54, 1474

Damour, T., \& Esposito-Farèse, G., 1998, Phys. Rev. D, 58, 042001

Freire, P. C. C., Wex, N., Esposito-Farèse, G., \& et al., 2012, MNRAS, 423, 3328

Fujii, Y., \& Maeda, K.-I., 2003, The Scalar-Tensor Theory of Gravitation, Cambridge Univ. Press

Ghez, A. M., Salim, S., Weinberg, N. N., \& et al., 2008, ApJ, 689, 1044

Gillessen, S., Eisenhauer, F., Trippe, S., \& et al., 2009, ApJ, 692, 1075

Hansen, R. O., 1974, J. Math. Phys., 15, 46

Liu, K. 2012, Exploring Pulsar-Black hole Binaries Using The Next Generation of Radio Telescopes, $\mathrm{PhD}$ thesis, University of Manchester

Liu, K., Wex, N., Kramer, M., Cordes, J. M., Lazio, T. J. W., ApJ, 747, 1

Liu, K., Eatough, R. P., Wex, N., Kramer, M., in prep.

Lorimer, D. R., \& Kramer, M., 2005, Handbook of Pulsar Astronomy, Cambridge Univ. Press

Penrose, R. 1979, in General Relativity: An Einstein Centenary Survey, Vol. 1, 581, ed. S. W.

Hawking \& W. Israel, Cambridge Univ. Press

\&Price, R. H., 1972a, Phys. Rev. D, 5, 2419

\&Price, R. H., 1972b, Phys. Rev. D, 5, 2439

Weisberg, J. M., Nice, D. J., \& Taylor, J. H., 2010, ApJ, 722, 1030

Wex, N. \& Kopeikin, S. 1999, ApJ, 514, 388

Will, C. M., 2008, ApJ, 674, L25 